\title{
Efficacy of SGLT2 inhibitor in type 2 diabetic patients under dietary instructions: A pilot study
}

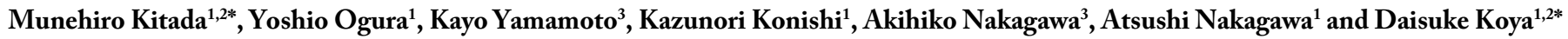 \\ ${ }^{1}$ Department of Diabetology and Endocrinology, Kanazawa Medical University, Japan \\ ${ }^{2}$ Division of Anticipatory Molecular Food Science and Technology, Medical Research Institute, Kanazawa Medical University, Uchinada, Ishikawa, Japan \\ ${ }^{3}$ Division of Clinical Nutrition, Kanazawa Medical University Hospital, Japan
}

\begin{abstract}
Aim: Insufficient adherence to diet therapy, or hyperphagia may decrease the effect on glycemic control by antidiabetic medicines, including sodium-glucose cotransporter 2 inhibitor (SGLT2i). We aimed to investigate the beneficial effects of SGLT2i, under dietary instructions, in type 2 diabetic (T2DM) patients.

Methods: Twenty-nine T2DM patients received SGLT2i for 48 weeks, and we analyzed the changes in metabolic parameters, including HbA1c and body composition. Diet was assessed with a validated brief-type self-administered diet history questionnaire (BDHQ), and the participants received dietary instructions. After the study, we followed 27 participants on the changes of HbA1c and body composition, for 96 weeks.

Results: Administration of SGLT2i significantly improved the metabolic parameters, including HbA1c and fat mass, compared to those of the baseline, throughout 48 weeks. Total energy intake and macronutrient balance showed no change throughout the study periods. Adherence to diet therapy was well maintained, and hyperphagia was also not shown. At week 72 and 96 after the end of the study, the HbA1c values were significantly elevated, compared to those at the end of the study.

Conclusions: Dietary instructions accompanied with a well adherence, is possibly necessary to exert the long-term stable beneficial effects with SGLT2i administration, in T2DM patients.
\end{abstract}

\section{Introduction}

Sodium-glucose cotransporter 2 inhibitor (SGLT2i) blocks the reabsorption of filtered glucose from the glomeruli, which are mainly located in renal proximal tubular cells. This action results in increased urinary glucose excretion and reduced blood glucose levels in type 2 diabetes mellitus (T2DM) patients [1]. SGLT2i can improve glucose control when used as both a monotherapy and additional therapy with other anti-diabetic agents, including metformin, dipeptidyl peptidase (DPP)-4 inhibitor, sulfonylurea (SU), a-glucosidase inhibitor ( $\alpha$ GI), glinide, pioglitazone or insulin [2-6]. In addition to having an anti-diabetic effect, SGLT2i has pleiotropic beneficial effects on the reduction of body weight (BW), blood pressure (BP), and uric acid (UA), as well as on the improvement of the lipid profile in T2DM patients $[7,8]$. Therefore, SGLT2 inhibitors may be a useful therapeutic strategy for preventing diabetic vascular complications. However, for some T2DM patients, the effects of SGLT2i on glycemic control or BW loss are limited during treatment with SGLT2i.

Diet therapy is fundamentally important for T2DM treatment. SGLT2i may increase dietary caloric (energy) intake, which is recognized as compensatory hyperphagia and has been determined to be the result of an adaptive response to calorie loss by increased urinary glucose excretion in animal models of T2DM [9-11]. The increased calorie intake that occurred after SGLT2i treatment may decrease the effects on the improvement of glycemic control or BW loss. A previous report also showed that SGLT2i treatment in T2DM patients induced compensatory hyperphagia based on energy balance dynamics calculated by a mathematical model [12]. Therefore, the BW loss induced by SGLT2i treatment may be lower than the expected BW loss amount calculated by calorie loss. In this study, we investigated the relationship between the effects of SGLT2i and changes in the amount of dietary intake, such as the total energy intake; energy ratio of nutrients, including carbohydrates, proteins and fat; and other specific nutrient intakes in T2DM patients under a diet therapy instructed by a dietitian. In addition, we followed the patients for 96 weeks after the first study, and evaluated the effect of frequent dietary instructions on glycemic control and body composition.

\section{Research design and methods}

\section{Subjects}

A total of 30 participants with T2DM (13 men and 17 women) were selected for the present study from patients who visited the

${ }^{\star}$ Correspondence to: Daisuke Koya, Department of Diabetology and Endocrinology, Kanazawa Medical University, Uchinada, Ishikawa, Japan, Tel: +81-76-286-221; Fax: +81-76-286-6927; E-mail: koya0516@kanazawa-med.ac.jp

Munehiro Kitada, Department of Diabetology and Endocrinology, Kanazawa Medical University, Uchinada, Ishikawa, Japan, Tel: +81-76-286-221; Fax: +8176-286-6927; E-mail: kitta@kanazawa-med.ac.jp

Key words: type 2 diabetes mellitus, SGLT2 inhibito, dietary instructions, body composition

Received: July 06, 2020; Accepted: July 13, 2020; Published: July 20, 2020 
Department of Endocrinology and Metabolism at Kanazawa Medical University Hospital. The inclusion criteria were (1) age $\geq 20$ years old and (2) T2DM. The exclusion criteria were (1) type 1 diabetes; (2) severe diabetic metabolic complications, such as ketoacidosis; (4) severe liver dysfunction; (5) hemodialysis; and (6) pregnant or nursing women and those who might be pregnant. All patients were deemed suitable by a physician to receive SGLT2 inhibitor. Additionally, a follow-up study was performed on the participants who were taken the informed consent. The participants were given detailed explanations of the study protocol, and informed consent was obtained from each patient. The study was conducted in accordance with the guidelines of the Declaration of Helsinki. The study protocol was registered with the University Hospital Medical Information Network (UMIN no. 000022738). The protocol of a follow-up study was approved by the Ethics Committee of Kanazawa Medical University (The ethics committee approval number: E 019).

\section{Study protocol}

The present study is a single-arm, open label, prospective and pilot study. After obtaining the subjects' informed consent, SGLT2i (100 $\mathrm{mg}$ canagliflozin, $5 \mathrm{mg}$ dapagliflozin, $2.5 \mathrm{mg}$ luseogliflozin, $50 \mathrm{mg}$ ipragliflozin or $10 \mathrm{mg}$ empagliflozin per day) was administered for 48 weeks in a clinical practice, in addition to other anti-diabetic agents. During the 48-week study period, changes in baseline medication use were allowed by reducing antidiabetic agents, including sulfonyl urea and insulin, for the prevention of hypoglycemia. The participants were assessed for the endpoint parameters at baseline and at 2, 4, 12, 24, 32, 40 and 48 weeks after additional treatment with SGLT2i. Additionally, a dietary assessment and dietary instructions by a dietitian was performed, as described below.

The primary endpoint of this study was defined as a change in HbA1c levels. The secondary endpoints included changes of body composition measured by In Body 720 (Biospace Japan, Inc., Tokyo, Japan), fasting plasma glucose (FPG), glycated albumin (GA), serum C-peptide (CPR), liver and renal function, UA, low-density lipoproteincholesterol (LDL-C), high-density lipoprotein-cholesterol (HDL-C), triglyceride (TG), hematocrit $(\mathrm{Ht})$, hemoglobin $(\mathrm{Hb})$, ketone bodies (total ketone body, 3-hydroxybutyrate (3-OHBA) and acetoacetic acid $(\mathrm{AcAc})$ ) and dietary assessment.

Additionally, we collected the data of the HbAlc values after 24, 48, 72 and 96 weeks, and body composition after 48, 72 and 96 weeks in 27 participants after the end of the study, as described below.

\section{Measurements}

Blood samples were collected in the morning after an overnight fast at baseline and at 4, 12,24,32, 40 and 48 weeks during the study periods (Figure 1A). The blood sample data were measured as previously described [13]. Serum CPR was measured by a chemiluminescent enzyme immunoassay method (Lumipulse Presto C-peptide, FUJREBIO INC., Tokyo, Japan). Serum total ketone bodies, 3-OHBA and AcAc levels were measured by enzymatic methods (Kainos 3-HB, total ketone, ketone body standard solution II, Kainos Laboratories, Inc., Tokyo, Japan). The data on ketone bodies including total ketone bodies, 3-OHBA and AcAc were log10-transformed before analysis because of their skewed distribution. The results of the analysis were back-transformed to obtain the geometric means of each ketone body of the observational-week value to the baseline value; the values then were expressed as fold changes in the adjusted geometric mean of each ketone body ratio of the observational-week value to the baseline value.
Additionally, we collected the HbAlc values after 24, 48, 72 and 96 weeks after the end of the study (Figure 1B).

Body composition was measured using In Body at 4, 12, 24, 32, 40 and 48 weeks during the study periods (Figure 1A), under the participants being lightly clothed, and also urinated the 30 minutes prior to the measurement of body composition. Additionally, we collected the data of fat mass (FM) from the results of body composition at 48, 72 and 96 weeks after the end of the study (Figure 1B).

\section{Dietary assessment}

Dietary intake during the preceding one month was assessed with a validated, self-administered, brief diet history questionnaire (BDHQ) $[14,15]$. The BDHQ requires only $15 \mathrm{~min}$ to complete; it is a 4-page fixed-portion questionnaire that asks about the consumption frequency of a total of 58 foods and beverages that are commonly consumed in the general Japanese population [16]. A dietitian supported the BDHQ by providing nutritional education for the participants before the start of the study and at $4,12,24,40$ and 48 weeks after the addition of SGLT2i (Figure 1A). Dietary intakes, in terms of energy and selected nutrients, were estimated by applying an ad hoc computer algorithm to the 58 foods and beverages of the BDHQ and to the Standard Tables of Food Composition in Japan. The mean daily intakes of total energy and 99 different nutrients can be estimated by the BDHQ. We also adjusted the basic index from the BDHQ, including total energy, protein, lipid, and carbohydrate intakes. The food-group intakes and nutrient intakes estimated by the BDHQ have been correlated with an assessment based on 16-day dietary records. The participants received an individual face-to-face nutritional instruction on diet therapy for diabetes by the dietitian for more than 30 minutes per one time at the beginning of the study and at 4, 12, 24, 32 and 40 weeks (Figure 1A).

\section{Statistical analysis}

The statistical analyses were performed with a StatMate 5 system (Abacus Concepts, Berkeley, CA, USA) for Windows. All results are expressed as the mean and standard deviation (SD). We used the repeated measure ANOVA with Dunnett's adjustment. Statistical significance was defined as $\mathrm{p}<0.05$.

\section{Results}

\section{Characteristics of the participants}

A total of 30 participants with T2DM were enrolled in this study. One individual did not complete the protocol due to the participant's wishes and not due to an adverse effect. Therefore, 29 participants completed the full protocol and were included in the statistical analyses. The participants' characteristics and all data at baseline are shown in Tables 1 and 2.

\section{Efficacy of an additional treatment with the SGLT2 inhibitor on glycemic control, lipid, liver and renal function tests, UA, levels of $\mathrm{Ht}$ and $\mathrm{Hb}$, and ketone bodies}

The values of HbAlc, FPG and GA were significantly reduced after 4 weeks of additional treatment with SGLT2i, and their reductions with SGLT2i treatment were maintained at 48 weeks compared to baseline (Table 2, Supplemental Figures 1A and 1B). The HbA1c levels exhibited a mean change of $-1.3 \%$ at the end of the study, which was significantly decreased compared to baseline (Figure 2A). SGLT2i significantly reduced the levels of serum CPR after 24 to 48 weeks compared to baseline (Table 2 and Supplemental Figure 1C). Glucosuria was 
A

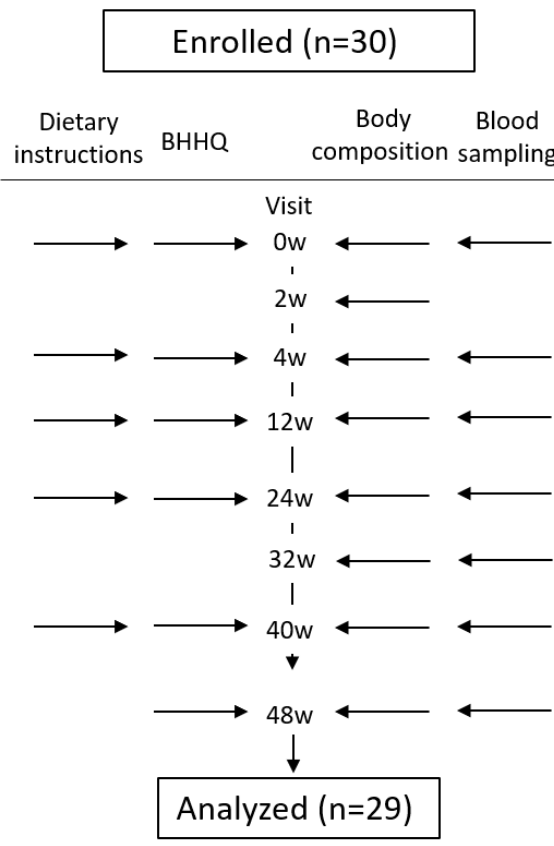

Discontinued SGLT2i treatment $(n=1)$

-Participant's wish (not adverse effect)

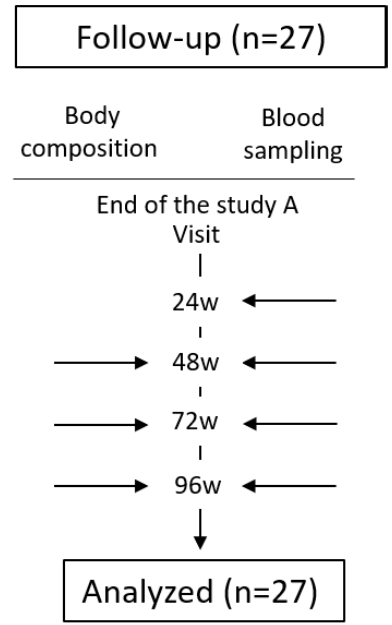

Figure 1. Flow diagram of the study

A. 30 participants were enrolled for this study. The participants received a dietary assessment by a validated brief-type self-administered diet history questionnaire (BDHQ) at $0,4,12,24$, 40 and 48 weeks, and dietary instructions by a dietitian at $0,4,12,24,40$ and 48 weeks. One participant discontinued the SGLT2 inhibitor treatment due to participant's wish (not adverse effect), and 29 participants completed the study and the results were analyzed. The participants also received the measurement of body composition and blood sampling at the indicated visit B. 27 individuals were followed for 96 weeks after the end of the study. Data of the HbAlc values and body composition were collected at the indicated visit, and the results were analyzed.

Table 1. Characteristics of participants

\begin{tabular}{|c|c|c|c|}
\hline n & 29 & Other medicines for hypertension or dyslipidemia & $\mathbf{n}$ \\
\hline Age (years old) & $55.5 \pm 10.2$ & Statin & 17 \\
\hline Male: Female & $12: 17$ & RAS inhibitor & 11 \\
\hline Body mass index $\left(\mathrm{kg} / \mathrm{m}^{2}\right)$ & $30.1 \pm 5.7$ & Ca-blocker & 9 \\
\hline Duration of diabetes (years) & $8.9 \pm 6.7$ & & \\
\hline Medication for diabetes & $\mathbf{N}$ & SGLT2 inhibitor & $\mathbf{n}$ \\
\hline DPP-4 inhibitor & 22 & Canagliflozin (100mg/day) & 7 \\
\hline Sulfonylurea & 9 & Luseogliflozin ( $2.5 \mathrm{mg} /$ day $)$ & 6 \\
\hline Metformin & 26 & Dapagliflozin (5mg/day) & 6 \\
\hline Insulin & 7 & Empagliflozin (10mg/day) & 5 \\
\hline Glinide & 1 & Ipragliflozin (50mg/day) & 5 \\
\hline Pioglitazon & 1 & & \\
\hline$\alpha$-glucosidase inhibitor & 2 & & \\
\hline
\end{tabular}

DPP-4, dipeptidyl peptidase-4; RAS, renin-angiotensin system; SGLT2, sodium glucose cotransporter 2

Results are expressed as the means \pm standard deviation (SD).

significantly increased by SGLT2i treatment during the study periods (Table 2). The levels of HDL-C were significantly elevated compared to 24 weeks after the treatment with SGLT2i (Table 2 and Figure 2B). However, LDL-C and TG levels showed no significant changes during the study periods (Table 2, Supplemental Figures 1D and $1 \mathrm{E})$. The data from the liver function tests including aspartate amino transferase (AST), alanine amino transferase (ALT) and $\gamma$-glutamyl transpeptidase $(\gamma$-GTP) indicated improvements after weeks 12 to 48 with additional SGLT2 i treatment (Table 2 and Figure 2C, Supplemental Figures $1 \mathrm{~F}$ and $1 \mathrm{G})$. The serum UA levels were significantly reduced from weeks 4 to 48 by SGLT2i treatment (Table 2 and Figure 2D). The eGFR levels did not change after treatment with SGLT2i during the study periods (Table 2, Supplemental Figure $1 \mathrm{H}$ ). The levels of $\mathrm{Ht}$ and $\mathrm{Hb}$ also exhibited significant elevations after treatment with SGLT2i (Table 2, Figure 2E and Supplemental Figure 1I). Additionally, log-transformed total ketone bodies showed a significant increase after 4 and 12 weeks of SGLT2i treatment (Table 2), and the fold change of log-transformed total ketone bodies, 3-OHBA and AcAc exhibited significant elevation compared to baseline during weeks 4 to 48 after additional treatment with SGLT2i (Figure 2F, Supplemental Figure 2J and 2K).

\section{Efficacy of additional treatment with the SGLT2 inhibitor on} body composition and blood pressure.

BW, FM and \% fat was significantly reduced after 4 weeks of additional SGLT2i treatment (Table 3). At the end of the study, the mean changes of BW, FM and $\%$ fat were $-4.1 \mathrm{~kg},-3.6 \mathrm{~kg}$ and $-2.7 \%$ compared to baseline, 
Table 2. Laboratory data, body composition and blood pressure before and after an additional treatment with SGLT2 inhibitor

\begin{tabular}{|c|c|c|c|c|c|c|c|}
\hline & 0 (Baseline) & 4 & 12 & 24 & 32 & 40 & 48 weeks \\
\hline \multicolumn{8}{|l|}{ Glucose metabolism related data } \\
\hline HbA1c (\%) & $8.4 \pm 1.6$ & $7.8 \pm 1.0^{\mathrm{a}}$ & $7.4 \pm 0.9^{* * *}$ & $7.2 \pm 0.7^{* * *}$ & $7.2 \pm 0.8^{* *}$ & $7.2 \pm 0.8^{* * *}$ & $7.1 \pm 0.6^{* *}$ \\
\hline FPG $(\mathrm{mg} / \mathrm{dl})$ & $157.6 \pm 46.9$ & $137.7 \pm 25.6^{* *}$ & $134.2 \pm 24.9^{* *}$ & $132.3 \pm 22.1^{* * *}$ & $129.5 \pm 22.0^{* * *}$ & $131.8 \pm 23.5^{* *}$ & $129.5 \pm 22.6^{* *}$ \\
\hline $\mathrm{GA}(\%)$ & $19.5 \pm 3.6$ & $16.7 \pm 2.5^{* * *}$ & $16.3 \pm 2.5^{* *}$ & $15.9 \pm 2.3^{* *}$ & $16.1 \pm 2.6^{* *}$ & $16.0 \pm 2.5^{* *}$ & $16.0 \pm 2.3^{* * *}$ \\
\hline CPR (ng/ml) & $2.26 \pm 1.10$ & $2.14 \pm 1.00$ & $2.11 \pm 1.00$ & $1.99 \pm 0.85^{*}$ & $1.91 \pm 0.82^{* *}$ & $1.99 \pm 0.90^{*}$ & $1.99 \pm 0.92^{*}$ \\
\hline Glucosuria (g/gCr) & $4.6 \pm 12.1$ & $55.0 \pm 34.5^{* * *}$ & $58.7 \pm 40.6^{* *}$ & $49.0 \pm 31.1^{* * *}$ & $43.5 \pm 25.0^{* *}$ & $44.4 \pm 26.9^{* *}$ & $49.7 \pm 29.8^{* * *}$ \\
\hline \multicolumn{8}{|l|}{ Lipid data } \\
\hline LDL-C (mg/dl) & $107.0 \pm 29.9$ & $103.9 \pm 29.6$ & $105.1 \pm 26.5$ & $105.8 \pm 20.7$ & $107.2 \pm 25.2$ & $103.0 \pm 27.8$ & $104.7 \pm 28.5$ \\
\hline HDL-C (mg/dl) & $45.9 \pm 11.2$ & $45.6 \pm 10.6$ & $47.3 \pm 12.0$ & $50.1 \pm 12.0^{* * *}$ & $50.6 \pm 12.4^{* *}$ & $49.9 \pm 12.1^{* *}$ & $50.7 \pm 12.4^{* * *}$ \\
\hline TG (mg/dl) & $128.2 \pm 76.0$ & $117.6 \pm 59.4$ & $113.7 \pm 102.1$ & $114.3 \pm 82.5$ & $123.4 \pm 97.0$ & $123.3 \pm 92.7$ & $120.7 \pm 86.6$ \\
\hline \multicolumn{8}{|l|}{ Liver function test } \\
\hline AST (IU/ml) & $36.6 \pm 20.7$ & $33.2 \pm 20.2$ & $28.5 \pm 14.1^{* *}$ & $25.9 \pm 12.8^{* *}$ & $24.7 \pm 16.5^{* *}$ & $25.0 \pm 14.5^{* *}$ & $24.4 \pm 11.5^{* *}$ \\
\hline ALT (IU/ml) & $55.1 \pm 37.7$ & $50.2 \pm 38.8$ & $39.7 \pm 27.5^{* *}$ & $35.3 \pm 25.7^{* *}$ & $31.4 \pm 25.7^{* *}$ & $32.1 \pm 27.2^{* *}$ & $32.1 \pm 21.7^{* * *}$ \\
\hline$\gamma$-GTP $(\mathrm{IU} / \mathrm{ml})$ & $59.4 \pm 58.1$ & $46.0 \pm 42.9^{* *}$ & $38.2 \pm 32.0^{* *}$ & $40.3 \pm 45.1^{* *}$ & $36.6 \pm 34.4^{* *}$ & $36.7 \pm 36.7^{* *}$ & $36.9 \pm 34.2^{* *}$ \\
\hline \multicolumn{8}{|l|}{ Uric acid and renal function test } \\
\hline $\mathrm{UA}(\mathrm{mg} / \mathrm{dl})$ & $5.3 \pm 1.0$ & $4.8 \pm 1.0^{* * *}$ & $4.8 \pm 1.0^{* *}$ & $4.8 \pm 0.9^{* * *}$ & $4.7 \pm 0.9^{* *}$ & $4.8 \pm 0.9^{* * *}$ & $4.7 \pm 0.8^{* *}$ \\
\hline $\begin{array}{l}\text { eGFR } \\
\left(\mathrm{ml} / \mathrm{min} / 1.73 \mathrm{~m}^{2}\right)\end{array}$ & $85.9 \pm 22.5$ & $83.7 \pm 22.4$ & $85.9 \pm 23.1$ & $84.6 \pm 20.2$ & $85.2 \pm 20.6$ & $84.6 \pm 22.7$ & $84.8 \pm 22.1$ \\
\hline \multicolumn{8}{|l|}{ Blood count test } \\
\hline Ht (\%) & $41.8 \pm 4.2$ & $42.8 \pm 4.6^{*}$ & $43.4 \pm 4.8^{* *}$ & $43.8 \pm 4.7^{* *}$ & $43.4 \pm 4.6^{* *}$ & $43.7 \pm 4.8^{* *}$ & $43.5 \pm 5.2^{* *}$ \\
\hline $\mathrm{Hb}(\mathrm{g} / \mathrm{dl})$ & $13.9 \pm 1.7$ & $14.1 \pm 1.7$ & $14.3 \pm 1.7^{* *}$ & $14.5 \pm 1.8^{* *}$ & $14.3 \pm 1.7^{* *}$ & $14.5 \pm 1.8^{* *}$ & $14.5 \pm 2.1^{* *}$ \\
\hline \multicolumn{8}{|l|}{ Ketone body } \\
\hline Total ketone body $(\mu \mathrm{mol} / \mathrm{l})$ & $165.7 \pm 122.6$ & $457.0 \pm 489.6^{* *}$ & $282.8 \pm 210.0$ & $165.7 \pm 122.6$ & N.E. & N.E. & $264.7 \pm 242.6$ \\
\hline Log-transformed total ketone body $(\mu \mathrm{mol} / \mathrm{l})$ & $2.13 \pm 0.28$ & $2.51 \pm 0.34^{* *}$ & $2.34 \pm 0.32^{*}$ & $2.29 \pm 0.30$ & N.E. & N.E. & $2.29 \pm 0.33$ \\
\hline $3-\mathrm{OHBA}(\mu \mathrm{mol} / \mathrm{l})$ & $110.8 \pm 92.9$ & $318.1 \pm 361.8^{* *}$ & $194.9 \pm 156.2^{*}$ & $110.8 \pm 92.9$ & N.E. & N.E. & $178.2 \pm 166.7$ \\
\hline Log-transformed 3-OHBA $(\mu \mathrm{mol} / \mathrm{l})$ & $1.93 \pm 0.31$ & $2.34 \pm 0.36^{* *}$ & $2.16 \pm 0.34^{*}$ & $2.10 \pm 0.34$ & N.E. & N.E. & $2.11 \pm 0.35$ \\
\hline $\operatorname{AcAc}(\mu \mathrm{mol} / \mathrm{l})$ & $54.9 \pm 31.8$ & $138.9 \pm 130.3^{* *}$ & $87.9 \pm 57.4$ & $54.9 \pm 31.8$ & N.E. & N.E. & $86.5 \pm 78.1$ \\
\hline Log-transformed AcAc $(\mu \mathrm{mol} / \mathrm{l})$ & $1.67 \pm 0.24$ & $2.10 \pm 0.33^{* *}$ & $1.85 \pm 0.29^{*}$ & $1.83 \pm 0.25$ & N.E. & N.E. & $1.82 \pm 0.31$ \\
\hline
\end{tabular}

Results are expressed as the means \pm standard deviation (SD). ${ }^{*}: \mathrm{p}<0.05$ vs. baseline, ${ }^{* * *}: \mathrm{p}<0.01$ vs. baseline, ANOVA with Dunnett's adjustment.

FPG, fasting plasma glucose; GA, glycated albumin, CPR C-peptide; LDL-C, low density lipoprotein-cholesterol; HDL-C, high density lipoprotein-cholesterol; TG, triglyceride,; AST, aspartate amino transferase; ALT, alanine amino transferase; $\gamma$-GTP, $\gamma$-glutamyl transpeptidase; UA, uric acid; eGFR, estimated glomerular filtration rate; Ht, hematocrit; Hb, hemoglobin; 3-OHBA, 3-hydroxybutyrate; AcAc, acetoacetate, N.E., not examined.

Table 3. Data on body composition and blood pressure before and after additional treatment with SGLT2 inhibitor

\begin{tabular}{|c|c|c|c|c|c|c|c|c|}
\hline & $\begin{array}{c}0 \\
\text { (Baseline) }\end{array}$ & 2 & 4 & 12 & 24 & 32 & 40 & 48 weeks \\
\hline \multicolumn{9}{|l|}{ Body composition } \\
\hline BW (kg) & $80.1 \pm 21.4$ & $78.6 \pm 21.4$ & $78.0 \pm 20.9^{*}$ & $76.8 \pm 21.1^{* *}$ & $75.9 \pm 20.7^{* * *}$ & $76.0 \pm 20.8^{* *}$ & $76.1 \pm 21.0^{* * *}$ & $76.0 \pm 20.6^{* *}$ \\
\hline FM (kg) & $30.5 \pm 12.3$ & $29.6 \pm 12.4$ & $29.1 \pm 12.0^{*}$ & $27.6 \pm 12.1^{* *}$ & $26.7 \pm 11.4^{* * *}$ & $26.6 \pm 11.3^{* *}$ & $27.0 \pm 11.2^{* *}$ & $26.9 \pm 11.3^{* * *}$ \\
\hline$\%$ Fat $(\%)$ & $37.2 \pm 7.5$ & $36.6 \pm 7.8$ & $36.4 \pm 7.4^{*}$ & $35.0 \pm 8.1^{* * *}$ & $34.4 \pm 7.4^{* * *}$ & $34.2 \pm 7.4^{* *}$ & $34.6 \pm 7.2^{* *}$ & $34.5 \pm 7.5^{* *}$ \\
\hline Skeletal Muscle (kg) & $27.3 \pm 7.2$ & $27.2 \pm 7.0$ & $26.9 \pm 7.0^{* *}$ & $27.0 \pm 7.1^{* * *}$ & $27.0 \pm 7.2^{*}$ & $27.1 \pm 7.1$ & $27.0 \pm 7.0^{*}$ & $27.0 \pm 7.1^{*}$ \\
\hline Intracellular Water (kg) & $22.5 \pm 5.5$ & $22.4 \pm 5.4$ & $22.2 \pm 5.4^{* * *}$ & $22.2 \pm 5.4^{*}$ & $22.2 \pm 5.5^{*}$ & $22.3 \pm 5.5$ & $22.2 \pm 5.4^{*}$ & $22.2 \pm 5.4^{*}$ \\
\hline \multicolumn{9}{|l|}{ Blood pressure } \\
\hline Systolic BP (mmHg) & $130.7 \pm 11.7$ & N.E. & $126.6 \pm 10.8$ & $122.2 \pm 13.5^{* *}$ & $122.3 \pm 8.8^{* * *}$ & $125.8 \pm 8.3$ & $123.3 \pm 9.1^{* * *}$ & $127.6 \pm 11.0$ \\
\hline Diastolic BP (mmHg) & $76.9 \pm 7.3$ & N.E. & $73.3 \pm 5.7^{*}$ & $71.8 \pm 3.8^{* *}$ & $73.1 \pm 5.6^{*}$ & $73.7 \pm 5.1^{*}$ & $74.6 \pm 5.5$ & $75.4 \pm 4.8$ \\
\hline
\end{tabular}

The results are expressed as the means \pm standard deviation (SD). ": $p<0.05$ vs. baseline, ${ }^{* *}: \mathrm{p}<0.01$ vs. baseline, ANOVA with Dunnett's adjustment. BW: Body weight, FM: Fat mass, BP: Blood pressure; N.E., not examined.

respectively (Table 3, Figure $2 \mathrm{G}$ and $2 \mathrm{H}$ ). Skeletal muscle mass (SMM) and intracellular water were decreased after treatment with SGLT2i (Table 3, Supplemental Figures 2A and B). Both systolic and diastolic BP were reduced after treatment with SGLT2i (Table 3, Figure 2I and Supplemental Figure 2C).

\section{Dietary assessment during the study period}

At baseline, the total energy intake was $1,717 \pm 551.1 \mathrm{kcal} /$ day $(29.5 \pm 9.1 \mathrm{kcal} / \mathrm{standard} \mathrm{BW} \mathrm{kg} /$ day $)$, and the energy proportions of carbohydrates (\%), protein and fat were $55.1 \pm 6.5 \%, 15.4 \pm 3.1 \%$ and $25.8 \pm 5.3 \%$, respectively (Figures $3 \mathrm{~A}$ and $3 \mathrm{~B}$ ). During the study periods, the total energy intake and energy ratio of macronutrients including carbohydrates, protein and fat showed no significant changes
(Figure 3B). Other nutritional intake amounts during the observational period, including sucrose, showed no significant change compared to baseline (Supplemental Table 1).

\section{Change of the HbAlc and FM after the end of the study}

We followed 27 participants who took the consent, and analyzed the change of the HbAlc and FM. The change amount of HbAlc was significantly elevated at 72 and 96 weeks from at end of the study ( $\mathrm{p}<$ 0.05 and $p<0.01$, respectively) (Figure $4 \mathrm{~A}$ ). The change of FM tended to increase, but not statistically significance, for 96 weeks after the end of the study (Figure 4B). At 96 weeks from the end of the study, the number of patients who showed the elevation of HbAlc was 19 (70.1\%), 

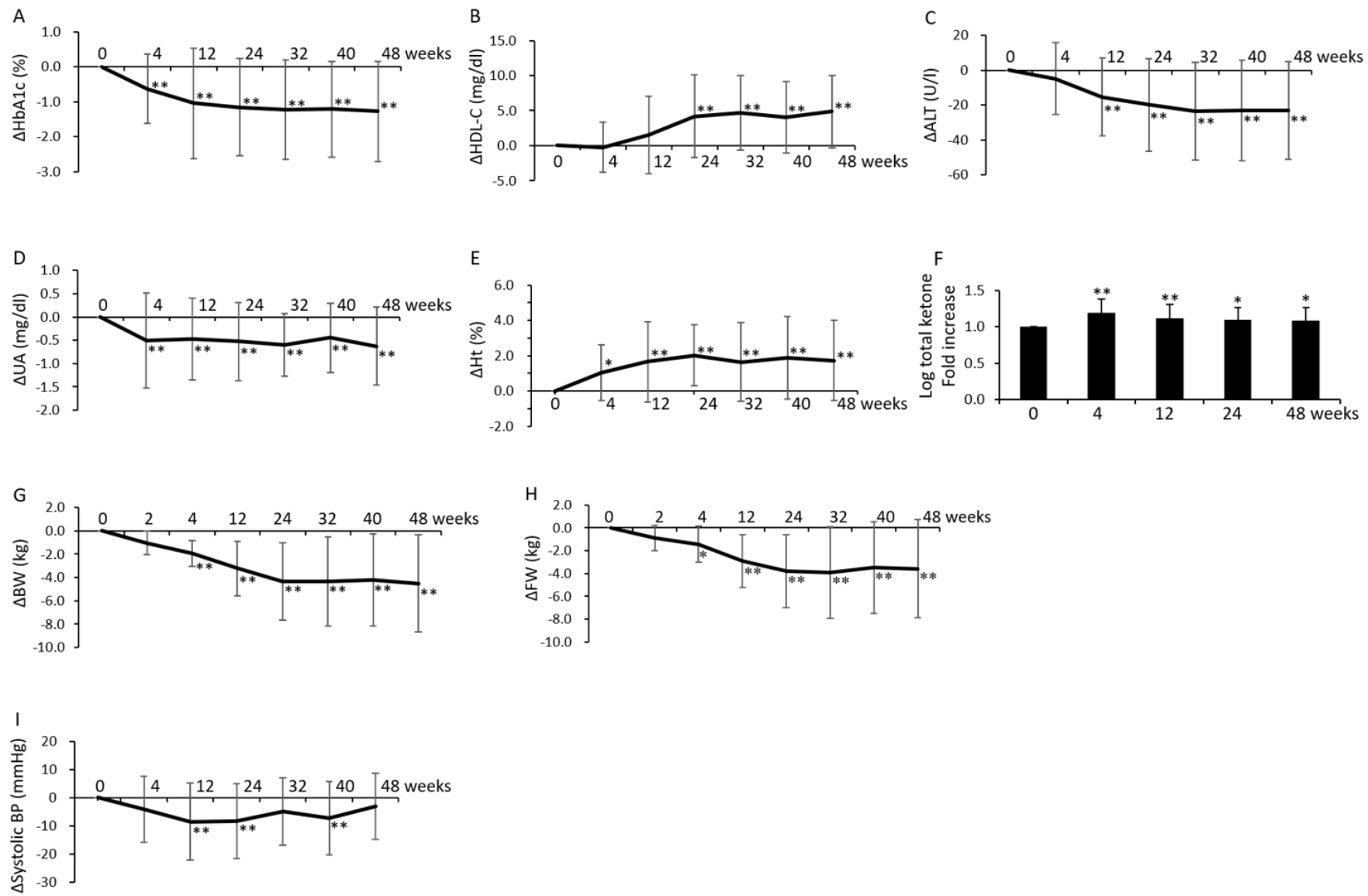

Figure 2. Changes in metabolic-related parameters and body composition during the study period

A. Changes in HbA1c, B. high-density lipoprotein-cholesterol (HDL-C), C. alanine transaminase (ALT), D. uric acid (UA), and E. hematocrit (Ht), from the baseline. F. Fold increase of log-transformed total ketone bodies compared to the baseline. G. Change in body weight (BW), H. fat mass (FM), and J. systolic blood pressure (BP) from the baseline.

Results are expressed as the means \pm standard deviation (SD). $\mathrm{n}=29,{ }^{*}: \mathrm{p}<0.05$ vs. baseline, ${ }^{* *}: \mathrm{p}<0.01$ vs. baseline

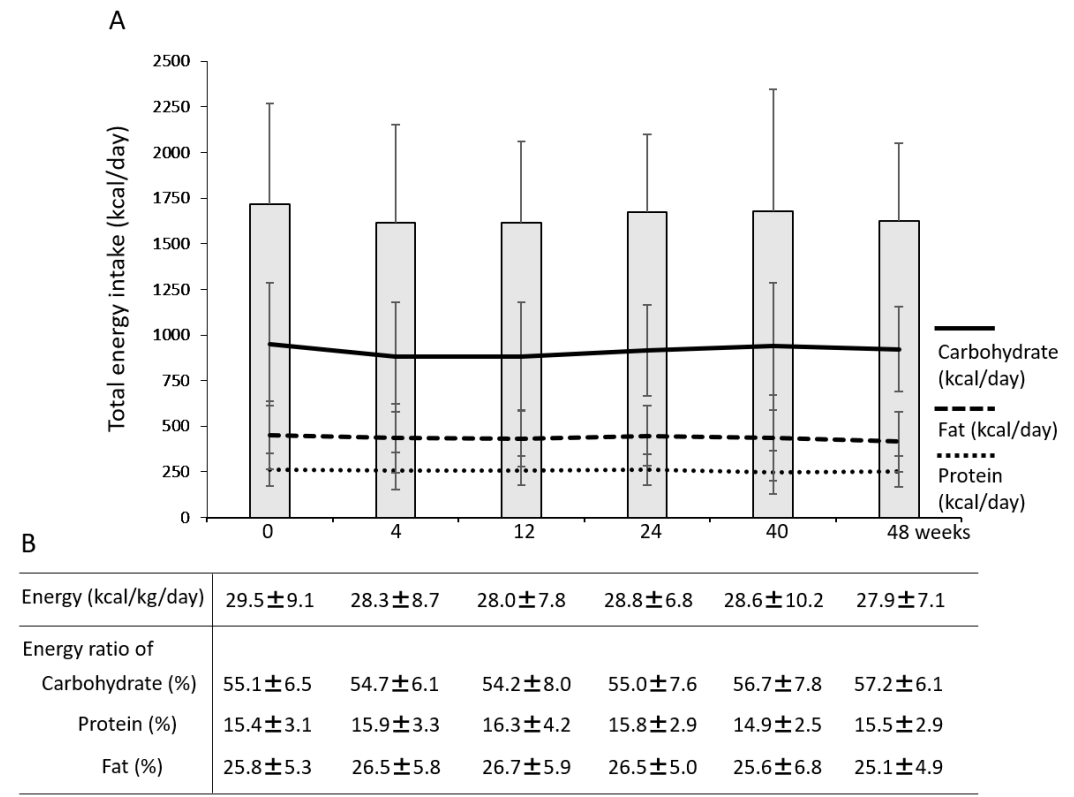

Figure 3. Dietary assessment and effect on change amount of HbAlc and FM

A. Total energy intake (kcal/day), carbohydrate intake ( $\mathrm{kcal} /$ day); solid line, protein intake (kcal/day); large dotted line, fat intake (kcal/day); small dotted line, during the study periods. B. Energy intake (kcal/standard body weight $(\mathrm{kg}) /$ day) and energy ratio of carbohydrates, protein and fat (\%) during the study periods.

Results are expressed as the means \pm standard deviation (SD), $\mathrm{n}=29$ 
A

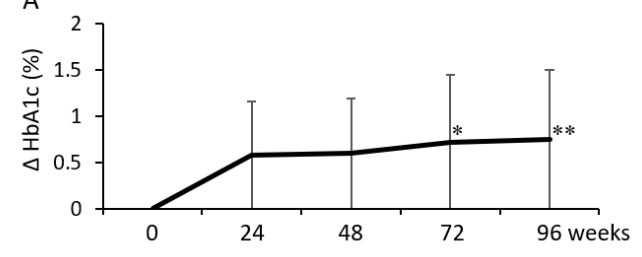

B

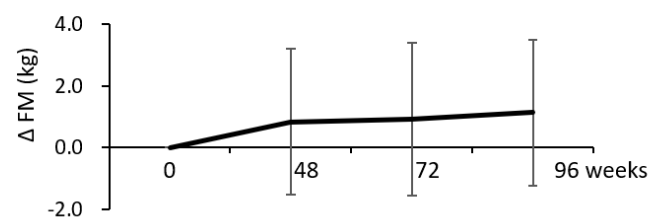

C

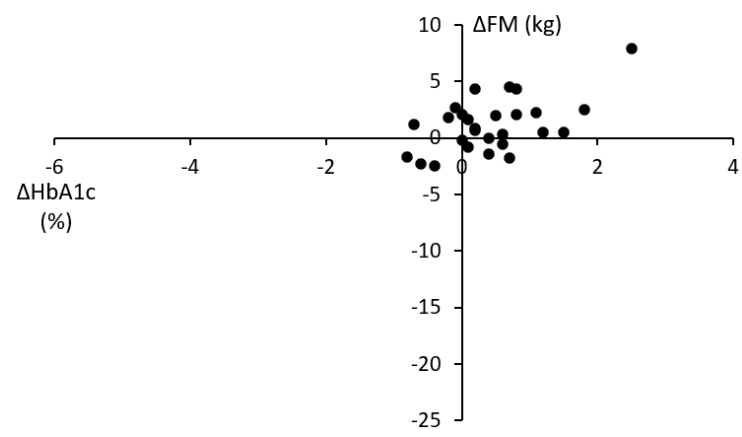

Figure 4. Change of the HbAlc and FM after the end of the study

A. Change in the value of $\mathrm{HbAlc}$ after the end of the study.

B. Change in the levels of FM after the end of the study.

C. Scatter plot; the change amount of HbA1c $(\triangle \mathrm{HbAlc})$ and FM $(\Delta \mathrm{FM})$ for 96 weeks from the end of the study.

Results are expressed as the means \pm standard deviation (SD). $n=27,{ }^{*}: \mathrm{p}<0.05$ vs. 0 week, ${ }^{* *}: \mathrm{p}<0.01$ vs. 0 week.

and 18 patients (66.7\%) showed the elevation of FM. In addition, $51.9 \%$ $(n=14)$ of patients exhibited the elevation of both HbAlc and FM (Figure 4C).

\section{Discussion}

In this pilot study, patients with T2DM who received additional treatment with SGLT2i clearly exhibited an improvement in glycemic control and a reduction in BW, mainly FM, for 48 weeks. Additionally, BP and other metabolic parameters, including HDL-C, UA and abnormal data of liver function tests, were improved after treatment with SGLT2i. Adherence to diet therapy, which was evaluated by the $\mathrm{BDHQ}$, was also preserved during the study periods. However, for 96 weeks after the end of the study, the patients had no frequent dietary instructions by a dietitian, consequently leading to the elevation of the HbA1c values.

Previous clinical reports have shown that SGLT2i improves glycemic control, lipid profile and non-alcoholic steatohepatitis (NASH), and decreases BW, BP and UA levels in T2DM patients [7,17-20]. Our results also showed a decrease in the mean change of HbAlc of $-1.3 \%$ after 48 weeks compared to baseline when used as an additional therapy to other anti-diabetic medicines. In addition to an increase of urinary glucose excretion, SGLT2i might improve glucose control through a decrease in insulin resistance, which is associated with a reduction in $\mathrm{FM}$, thereby resulting in the reduction of serum CPR levels. Additionally, elevated HDL-C levels, reduced UA levels and a slight reduction in BP were shown after additional treatment with SGLT2i. Moreover, previous reports have demonstrated that treatment with SGLT2i leads to increased serum ketone bodies [21], which may be associated with cardio-renal tissue protection [22]. Our data also showed that total ketone bodies, 3-OHBA and AcAc exhibited significant elevation, expressed as fold changes, from baseline to 4 to 48 weeks, after additional treatment with SGLT2i.
In addition to a glucose lowering effect, previous reports have demonstrated that SGLT2i reduced $\mathrm{BW}^{7,19,20}$, and most of the weight loss was from adipose tissue [23]. Our results on body composition measured by an impedance method using In Body indicated that SGLT2i significantly reduced the mean BW and FM compared to baseline. We also observed that the SMM and intracellular water were slightly but significantly decreased after SGLT2i treatment. Since a change in SMM showed a similar pattern to intracellular water, this suggests that the muscle related change was affected by changes in the body water content. The impedance method calculates the lean body mass (LBM), including SMM, by combining body water, protein and mineral content without bone components, which implies that a loss of body water may be reflected as a decrease in SMM. Several reports showed that SGLT2i treatment might be considered to reduce the LBM, including muscle volume in T2DM patients [23-25], and sarcopenia is a loss of muscle, resulting in increased frailty and mortality, particularly in elderly people [26]. Therefore, a careful observation regarding loss of muscle mass is necessary after treatment with SGLT2i. However, the muscle quality is also important. Sano et al. reported that grip strength significantly increased after 4 weeks of SGLT2i treatment in T2DM patients [27]. The long-term effects and the mechanisms on muscle quality during SGLT2i treatment are unclear; however, in addition to muscle volume, the quality of the muscle should be assessed after SGLT2i treatment.

Previous animal studies showed that compensatory calorie intake might cancel the BW reduction by SGLT2i-induced calorie loss [9-11]. Additionally, in patients with T2DM, SGLT2i treatment was shown to induce compensatory hyperphagia based on energy balance dynamics calculated by a mathematical model [12-28]. However, there are few reports regarding whether compensatory energy intake occurs after SGLT2i treatment in T2DM patients in the clinical setting. Yamamoto et al. [29] reported that ipragliflozin treatment in T2DM patients for 16 weeks decreased visceral adipose tissue and improved parameters for 
metabolic dysfunction, and the change in visceral adipose tissue (VAT) was only correlated to adherence of the diet therapy. These results suggest that the effects of SGLT2i on VAT may be affected by the change of energy intake. In addition, Matsuba et al. [30] demonstrated that after patients with T2DM treated with canagliflozin, their calorie intake increased without changing the ratio of the three macronutrients. In this study, the total energy intake evaluated by the BDHQ was not changed after additional treatment with SGLT2i, during the study periods for 48 weeks. The energy ratio of carbohydrates, protein and fat was also not significantly changed throughout the study periods. Thus, adherence for a diet therapy was maintained during the observational periods in this study. Previously, Horie et al. [15] reported that dapagliflozin treatment in T2DM patients showed no change in total calorie intake and proportions of carbohydrate, protein and fat for 3 months, but the intake of sugar was significantly increased in patients treated with dapagliflozin. In this study, although the intake of sucrose tended to decrease after 4 weeks and tended to increase again after 24 weeks, it did not change significantly from baseline throughout the study periods after SGLT2i administration. However, after the study, the $\mathrm{HbA1c}$ levels were elevated possibly due to not under frequent dietary instructions by a dietitian. Thus, a daily diet therapy is important for exerting the effects of SGLT2i on metabolic benefits such as glycemic control in T2DM patients for a long time. The frequent dietary instructions by a dietitian may play a crucial role for continuation of an adequate diet therapy for a long time. Serum ketone bodies exhibited the increased from the baseline through the study; therefore, a strict low carbohydrate diet, with a carbohydrate energy ratio lower than $40 \%$, should be avoided to prevent SGLT2 inhibitor-associated diabetic ketoacidosis [31].

There are several limitations in this study: 1) this study is a singlearm, open-label and pilot study; 2) the absence of control group without dietary instructions; 3) the sample size is small; 4) the BDHQ, a self-reported dietary assessment method, is subject to random and systematic measurement errors and the misreporting of dietary intakes, particularly by overweight/obese individuals, which is also a limitation associated with such self-reported dietary assessment methods [32]; 5) exercise and energy expenditure were not evaluated in this study; and 6) we did not evaluate an adherence of diet therapy and dietary intake using the BDHQ in a follow-up study.

\section{Conclusions}

SGLT2i displays significant metabolic benefits, including the improvement of glycemic control and the reduction of FM, however, dietary instructions accompanied with a well adherence, is possibly necessary to exert the long-term stable beneficial effects with SGLT2i, in T2DM patients. In addition, the frequent dietary instructions by a dietitian may play a crucial role for a diet therapy for T2DM patients. However, to clarify whether dietary instructions are necessary to exert the beneficial effects of SGLT2i in T2DM patients, further a large study comparing between dietary instructions intervention group and control group is necessary.

\section{Acknowledgments}

We thank Yuka Kuroshima, who are clinical research coordinators, and Eriko Kidani and Yukiho Kanamori-Kasahara and all the staff, in the Department of Endocrinology and Metabolism of Kanazawa Medical University Hospital, for their great assistance in this study.

\section{Authorship confirmation statement}

All authors are in agreement with the content of the manuscript. MK and DK designed the study, researched and analyzed the data, and wrote and edited the manuscript. MK, YO, KK, AN and DK contributed to the research and to the collection of the data. MK analyzed the data. KY and AN collected and analyzed the data of the BDHQ. KK contributed to the discussion. MK and DK are the guarantors of this work.

\section{Author disclosure statement}

The authors declare that there is no conflict of interest associated with this manuscript.

\section{Funding statement}

Boehringer Ingelheim, Mitsubishi Tanabe Pharma, Kyowa Kirin, Taisho Pharmaceutical Co. and Ono Pharmaceutical Co. contributed to establishing the Division of Anticipatory Molecular Food Science and Technology. The authors declare that there is no conflict of interest associated with this manuscript.

\section{References}

1. Chao EC, Henry RR (2010) SGLT2 inhibition--a novel strategy for diabetes treatment Nat Rev Drug Discov 9: 551-599. [Crossref]

2. Araki E, Tanizawa Y, Tanaka Y (2015) Long-term treatment with empagliflozin as add-on to oral antidiabetes therapy in japanese patients with type 2 diabetes mellitus. Diabetes Obes Metab 17: 665-674. [Crossref]

3. Cho YK, Kang YM, Lee SE (2018) Efficacy and safety of combination therapy with SGLT2 and DPP4 inhibitors in the treatment of type 2 diabetes: A systematic review and meta-analysis. Diabetes Metab 44: 393-401. [Crossref]

4. Molugulu N, Yee LS, Ye YT (2017) Systematic review of metformin monotherapy and dual therapy with sodium glucose co-transporter 2 inhibitor (SGLT-2) in treatment of type 2 diabetes mellitus. Diabetes Res Clin Pract 132: 157-168. [Crossref]

5. Yang Y, Chen S, Pan H (2017) Safety and efficiency of SGLT2 inhibitor combining with insulin in subjects with diabetes: Systematic review and meta-analysis of randomized controlled trials. Medicine (Baltimore) 96: e6944. [Crossref]

6. Rosenstock J, Vico M, Wei L (2012) Effects of dapagliflozin, an SGLT2 inhibitor, on $\mathrm{HbA}(1 \mathrm{c})$, body weight, and hypoglycemia risk in patients with type 2 diabetes inadequately controlled on pioglitazone monotherapy. Diabetes Care 35: 1473-1478. [Crossref]

7. Zinman B, Wanner C, Lachin JM (2015) Empagliflozin, cardiovascular outcomes, and mortality in type 2 diabetes. $N$ Engl J Med 373: 2117-2128. [Crossref]

8. Zaccardi F, Webb DR, Htike ZZ (2016) Efficacy and safety of sodium-glucose cotransporter-2 inhibitors in type 2 diabetes mellitus: systematic review and network meta-analysis. Diabetes Obes Metab 18: 783-794. [Crossref]

9. Devenny JJ, Godonis HE, Harvey SJ (2012) Weight loss induced by chronic dapagliflozin treatment is attenuated by compensatory hyperphagia in diet-induced obese (DIO) rats. Obesity (Silver Spring) 20: 1645-1652. [Crossref]

10. Nagata T, Fukuzawa T, Takeda M (2013) Tofogliflozin, a novel sodium-glucose cotransporter 2 inhibitor, improves renal and pancreatic function in $\mathrm{db} / \mathrm{db}$ mice. $\mathrm{Br} J$ Pharmacol 170: 519-531. [Crossref]

11. Iuchi H, Sakamoto M, Matsutani D (2017) Time-dependent effects of ipragliflozin on behaviour and energy homeostasis in normal and type 2 diabetic rats: continuous glucose telemetry analysis. Sci Rep 7: 11906.

12. Ferrannini G, Hach T, Crowe S (2015) Energy balance after sodium-glucose cotransporter 2 inhibition. Diabetes Care 38: 1730-1735. [Crossref]

13. Matsuo S, Imai E, Horio M (2009) Revised equations for estimated GFR from serum creatinine in Japan. Am J Kidney Dis 53: 982-992. [Crossref]

14. Sasaki S, Yanagibori R, Amano K (1998) Self-administered diet history questionnaire developed for health education: a relative validation of the test-version by comparison with 3-day diet record in women. J Epidemiol 8: 203-215. [Crossref]

15. Horie I, Abiru N, Hongo R (2018) Increased sugar intake as a form of compensatory hyperphagia in patients with type 2 diabetes under dapagliflozin treatment. Diabetes Res Clin Pract 135: 178-184. [Crossref] 
16. Kobayashi S, Murakami K, Sasaki S (2011) Comparison of relative validity of food group intakes estimated by comprehensive and brief-type self-administered diet history questionnaires against $16 \mathrm{~d}$ dietary records in Japanese adults. Public Health Nutr 14: 1200-1211. [Crossref]

17. Ferrannini E, Ramos SJ, Salsali A (2010) Dapagliflozin monotherapy in type 2 diabetic patients with inadequate glycemic control by diet and exercise: a randomized, doubleblind, placebo-controlled, phase 3 trial. Diabetes Care 33: 2217-2224. [Crossref]

18. Inagaki N, Kondo K, Yoshinari T (2013) Efficacy and safety of canagliflozin in Japanese patients with type 2 diabetes: a randomized, double-blind, placebo-controlled, 12-week study. Diabetes Obes Metab 15: 1136-1145. [Crossref]

19. Stenlof K, Cefalu WT, Kim KA (2013) Efficacy and safety of canagliflozin monotherapy in subjects with type 2 diabetes mellitus inadequately controlled with diet and exercise. Diabetes Obes Metab 15: 372-382. [Crossref]

20. Neal B, Perkovic V, Mahaffey KW (2017) Canagliflozin and cardiovascular and renal events in type 2 diabetes. $N$ Engl J Med 377: 644-657. [Crossref]

21. Min SH, Oh TJ, Baek SI (2018) Degree of ketonaemia and its association with insulin resistance after dapagliflozin treatment in type 2 diabetes. Diabetes Metab 44: 73-76. [Crossref]

22. Mudaliar S, Alloju S, Henry RR (2016) Can a shift in fuel energetics explain the beneficial cardiorenal outcomes in the EMPA-REG OUTCOME Study? A unifying hypothesis. Diabetes Care 39: 1115-1122. [Crossref]

23. Bolinder J, Ljunggren O, Johansson L (2014) Dapagliflozin maintains glycaemic control while reducing weight and body fat mass over 2 years in patients with type 2 diabetes mellitus inadequately controlled on metformin. Diabetes Obes Metab 16: 159-169. [Crossref]
24. Cefalu WT, Leiter LA, Yoon KH (2013) Efficacy and safety of canagliflozin versus glimepiride in patients with type 2 diabetes inadequately controlled with metformin (CANTATA-SU): 52 week results from a randomised, double-blind, phase 3 noninferiority trial. Lancet 382: 941-950. [Crossref]

25. Bolinder J, Ljunggren O, Kullberg J (2012) Effects of dapagliflozin on body weight, total fat mass, and regional adipose tissue distribution in patients with type 2 diabetes mellitus with inadequate glycemic control on metformin. J Clin Endocrinol Metab 97 1020-1031. [Crossref]

26. Yanase T, Yanagita I, Muta K (2018) Frailty in elderly diabetes patients. Endocr J 65: 1-11. [Crossref]

27. Sano M, Meguro S, Kawai T (2016) Increased grip strength with sodium-glucose cotransporter 2. J Diabetes 8: 736-737. [Crossref]

28. Rajeev SP, Cuthbertson DJ, Wilding JP (2016) Energy balance and metabolic changes with sodium-glucose co-transporter 2 inhibition. Diabetes Obes Metab 18: 125-134 [Crossref]

29. Yamamoto C, Miyoshi H, Ono K (2016) Ipragliflozin effectively reduced visceral fa in Japanese patients with type 2 diabetes under adequate diet therapy. Endocr $J$ 63: 589-596. [Crossref]

30. Matsuba I, KanamoriA, Takihata M (2020) Canagliflozin increases calorie intake in type 2 diabetes without changing the energy ratio of the three macronutrients: CANA-K Study. Diabetes Technol Ther 22: 228-234. [Crossref]

31. Yabe D, Iwasaki M, Kuwata H (2017) Sodium-glucose co-transporter-2 inhibitor use and dietary carbohydrate intake in Japanese individuals with type 2 diabetes: A randomized, open-label, 3-arm parallel comparative, exploratory study. Diabetes Obes Metab 19: 739-743. [Crossref]

32. Livingstone MB, Black AE (2003) Markers of the validity of reported energy intake. $J$ Nutr 133: 895S-920S. [Crossref]

Copyright: $\odot 2020$ Kitada M. This is an open-access article distributed under the terms of the Creative Commons Attribution License, which permits unrestricted use, distribution, and reproduction in any medium, provided the original author and source are credited. 